\title{
Rationalising medications through deprescribing
}

\author{
Careful judgment is required to optimise benefit and minimise harm
}

\author{
Anthony $\mathrm{J}$ Avery professor of primary healthcare, Brian G Bell research fellow \\ Division of Primary Care, School of Medicine, University of Nottingham, Nottingham, UK
}

With an ageing population and growth in multimorbidity and polypharmacy, increasing attention is being paid to deprescribing. This term has become widely used in recent years, and there are numerous definitions. ${ }^{1}$ Put simply, it is the process of withdrawing drugs to try to improve outcomes. ${ }^{2}$ But it is important to recognise this is a complex process; careful judgment is required to balance the potential risks and benefits of withdrawing medicines.

Since TheBMJ last published an editorial on deprescribing in $2014^{2}$ at least three systematic reviews have been published ${ }^{3-5}$ as well as several studies not included in these reviews. ${ }^{6-8}$ Here we consider the latest evidence and guidance on withdrawing drug treatments.

Clearly, patients should receive only the medications that they want and need. If deprescribing is required it's important to know the safest and most effective approach, what resources are needed to do it properly, and the likely ratio of costs to benefits for patients and health services.

Multiple studies of polypharmacy or deprescribing have shown that interventions by pharmacists, doctors, or multidisciplinary teams can reduce the number of medications that patients take and reduce the prevalence of potentially inappropriate prescribing. ${ }^{5}$ These reductions tend to be modest, however. For example, in one meta-analysis patients were taking an average of 7.4 drugs at baseline; during follow-up this fell by 0.2 drugs in intervention groups and increased by 0.2 drugs in control groups.

\section{Modest effects}

Research on clinical outcomes is also growing. Overall, deprescribing seems to be safe, but the evidence on benefits remains mixed. ${ }^{3-5}$ A meta-analysis of randomised trials showed no overall reduction in mortality from deprescribing interventions, ${ }^{4}$ although a subgroup analysis confined to interventions targeted at patients (rather than education for clinicians) did suggest a mortality benefit (odds ratio $0.62,95 \%$ confidence intervals 0.43 to 0.88 , relative to controls). In another meta-analysis, strategies to reduce polypharmacy did not reduce mortality ${ }^{3}$ or hospital admissions. At least 15 studies have examined the effects of deprescribing on quality of life, and only one of these reported a benefit (which was modest). ${ }^{4}$
Reliable cost effectiveness data are scarce. Although reducing prescribing, particularly hazardous prescribing, might be expected to save money, these savings must be offset against the cost of the deprescribing intervention. One recent analysis ${ }^{9}$ suggests that interventions to reduce polypharmacy are cost saving overall, but more research is needed to identify the most cost effective strategies and their key elements. ${ }^{10}$

The available guidance and published evaluations highlight that, done properly, deprescribing is complex and time consuming. When patients are taking multiple drugs, each must be carefully considered in the context of likely benefits and harms, therapeutic indications, potential drug interactions, the preferences of patients and carers, and adherence. A Scottish study estimated that this takes up to 30 minutes for a doctor and 75 minutes for a pharmacist. ${ }^{9}$ Health services should be under no illusion that benefits can be achieved in the single short consultations typical of general practice appointments in the UK. Investment is required to fund pharmacists, doctors, or multidisciplinary teams to do this work.

Considerable advice is available on deprescribing. Guidance from the Scottish Government Polypharmacy Model of Care Group is particularly helpful, offering a seven step process for reviewing medications. ${ }^{10}$ Key aspects include a strong focus on what matters to each patient and an emphasis on empowering and supporting patients in their decision making around medicines. Although there is some uncertainty whether shared decision making improves outcomes, ${ }^{11}$ a European consortium has made a strong case for informed discussions and shared decisions in deprescribing interventions for patients taking multiple drugs. ${ }^{12}$ There is a strong ethical argument too: respecting autonomy demands detailed discussion of the expected benefits and risks of all medicines, recognising that these can change over time, particularly with age or increasing frailty.

More research is needed to help identify the best approaches to deprescribing, and it's important to do this in collaboration with patients. Nevertheless, based on reasonably substantive evidence so far, it is unlikely that we are going to see major breakthroughs in this area. Probably the best we can expect is modest reductions in medication load and hazardous prescribing, and modest improvements in patient outcomes. Given the complexity 
of the task, overall cost savings are also likely to be modest. Deprescribing remains a worthwhile investment, however, and should be done in partnership with the patients and families who cope every day with burdensome polypharmacy.

Competing interests: The BMJ has judged that there are no disqualifying financial ties to commercial companies. The authors declare no other interests. Further details of The BMSs policy on financial interests is here: https://www.bmj.com/ sites/default/files/attachments/resources/2016/03/16-current-bmj-education-coiform.pdf.

Provenance and peer review: Commissioned; not externally peer reviewed.

1 Reeve E, Gnjidic D, Long J, Hilmer S. A systematic review of the emerging definition of 'deprescribing' with network analysis: implications for future research and clinical practice. Br J Clin Pharmacol 2015;80:1254-68. 10.1111/bcp.12732 27006985

2 Gnjidic D, Le Couteur DG, Hilmer SN. Discontinuing drug treatments. BMJ 2014;349:g7013 10.1136/bmj.g7013 25416561

3 Johansson T, Abuzahra ME, Keller S, etal . Impact of strategies to reduce polypharmacy on clinically relevant endpoints: a systematic review and meta-analysis. Br J Clin Pharmacol 2016;82:532-48. 10.1111/bcp.12959 27059768
4 Page AT, Clifford RM, Potter K, Schwartz D, Etherton-Beer CD. The feasibility and effect of deprescribing in older adults on mortality and health: a systematic review and meta-analysis. Br J Clin Pharmacol 2016;82:583-623. 10.1111/bcp.12975 2707723

5 Thillainadesan J, Gnjidic D, Green S, Hilmer SN. Impact of deprescribing interventions in older hospitalised patients on prescribing and clinical outcomes: A systematic review of randomised trials. Drugs Aging 2018;35:303-19. 10.1007/s40266-018-0536-4 29541966

6 Brodaty $\mathrm{H}$, Aerts L, Harrison F, etal . Antipsychotic deprescription for older adults in long-term care: The HALT study. J Am Med Dir Assoc 2018;19:592-600.e7. 10.1016/.jamda.2018.05.002 29941156

7 Westbury JL, Gee P, Ling T, etal . RedUSe: reducing antipsychotic and benzodiazepine prescribing in residential aged care facilities. Med J Aust 2018;208:398-403. 10.5694/mja17.00857 29747564

8 Wouters $\mathrm{H}$, Scheper J, Koning $\mathrm{H}$, etal . Discontinuing inappropriate medication use in nursing home residents: A cluster randomized controlled trial. Ann Intern Med 2017; 167:609-17. 10.7326/M16-2729 29052691

9 Michael N, Wiese B, The SIMPATHY consortium. The SIMPATHY economic analysis tool: user guide. $2017 \mathrm{http}: / / \mathrm{www}$. simpathy.eu/resources/change-management

10 Scottish Government Polypharmacy Model of Care Group. Polypharmacy guidance, realistic prescribing. 3rd ed. Scottish Government, 2018.

11 Légaré F, Adekpedjou R, Stacey D, etal . Interventions for increasing the use of shared decision making by healthcare professionals. Cochrane Database Syst Rev 2018;7:CD006732. 10.1002/14651858.CD006732.pub4. 30025154

12 Mair A, Fernandez-Llimos F, Alonso A, etal. The SIMPATHY Consortium. Polypharmacy management by 2030: a patient safety challenge. SIMPATHY Consortium, 2017.

Published by the BMJ Publishing Group Limited. For permission to use (where not already granted under a licence) please go to http://group.bmj.com/group/rights-licensing/ permissions 